University of Texas at El Paso

ScholarWorks@UTEP

4-2015

\title{
Model Reduction: Why It Is Possible And How It Can Potentially Help To Control Swarms Of Unmanned Arial Vehicles (UAVs)
}

\author{
Martine Ceberio \\ The University of Texas at El Paso, mceberio@utep.edu \\ Leobardo Valera \\ The University of Texas at El Paso, leobardovalera@gmail.com \\ Olga Kosheleva \\ The University of Texas at El Paso, olgak@utep.edu \\ Rodrigo A. Romero \\ The University of Texas at El Paso, raromero2@utep.edu \\ Follow this and additional works at: https://scholarworks.utep.edu/cs_techrep \\ Part of the Computer Engineering Commons \\ Comments: \\ Technical Report: UTEP-CS-15-12a \\ To appear in Proceedings of the Annual Conference of the North American Fuzzy Information \\ Processing Society NAFIPS'2015 and 5th World Conference on Soft Computing, Redmond, \\ Washington, August 17-19, 2015.
}

\section{Recommended Citation}

Ceberio, Martine; Valera, Leobardo; Kosheleva, Olga; and Romero, Rodrigo A., "Model Reduction: Why It Is Possible And How It Can Potentially Help To Control Swarms Of Unmanned Arial Vehicles (UAVs)" (2015). Departmental Technical Reports (CS). 911.

https://scholarworks.utep.edu/cs_techrep/911

This Article is brought to you for free and open access by the Computer Science at ScholarWorks@UTEP. It has been accepted for inclusion in Departmental Technical Reports (CS) by an authorized administrator of ScholarWorks@UTEP. For more information, please contact Iweber@utep.edu. 


\title{
Model Reduction: Why It Is Possible and How It Can Potentially Help to Control Swarms of Unmanned Arial Vehicles (UAVs)
}

\author{
Martine Ceberio, Leobardo Valera, \\ Olga Kosheleva, and Rodrigo Romero \\ University of Texas at El Paso \\ $500 \mathrm{~W}$. University \\ El Paso, TX 79968, USA \\ mceberio@utep.edu, leobardovalera@gmail.com \\ olgak@utep.edu, raromero2@utep.edu
}

\begin{abstract}
In many application areas, such as meteorology, traffic control, etc., it is desirable to employ swarms of Unmanned Arial Vehicles (UAVs) to provide us with a good picture of the changing situation and thus, to help us make better predictions (and make better decisions based on these predictions). To avoid duplication, interference, and collisions, UAVs must coordinate their trajectories. As a result, the optimal control of each of these UAVs should depend on the positions and velocities of all others - which makes the corresponding control problem very complicated. Since, in contrast to controlling a single UAV, the resulting problem is too complicated to expect an explicit solution, a natural idea is to extra expert rules and use fuzzy control methodology to translate these rules into a precise control strategy. However, with many possible combinations of variables, it is not possible to elicit that many rules.
\end{abstract}

In this paper, we show that, in general, it is possible to use model reduction techniques to decrease the number of questions and thus, to make rules elicitation possible. In addition to general results, we also show that for the UAVs, optimal control indeed leads to a model reduction - and thus, to a drastic potential decrease in the corresponding number of questions.

\section{SWARMS OF UNMANNED AERIAL VeHICles (UAVS): Potential Advantages AND CHALlEnges}

Case study: meteorology. Let us start our explanation of need for UAVs with meteorology.

One of the main objectives of meteorology is to predict future weather.

Specifics of meteorology: dynamic equations are known. The equations describing atmosphere are well known. Thus, in principle, if we know the initial state of the atmosphere, we can solve these equations and predict its future state.

Accurate long-term predictions are not possible, but shortterm predictions are possible. Of course, it is well known that long-term predictions cannot be accurate: due to the nature of the corresponding equations, a very small difference in initial conditions eventually gets drastically amplified, as result of which to get an accurate long-term prediction, we need an impossible accurate knowledge of initial conditions. This fact was first noticed by Edward N. Lorenz [20], who famously remarked that a flap of a butterfly's wing in Brazil can eventually cause a tornado in Texas; his results started what is now known as chaos theory.

However, for short-term predictions, the equations work really well.

Meteorological predictions: successes. The ability to use high performance computers to perform the corresponding predictions has made current weather predictions much more accurate than in the past.

Meteorological predictions: limitations. However, the prediction accuracy is still far from perfect. The main reason for this imperfection is that there are many areas of the Earth from which we do not have enough data about the current state of the atmosphere. Mostly, these are areas with few people living, such as desert areas, Arctic and Antarctic areas, etc.

How to overcome these limitations. To improve the prediction accuracy, it is important to measure the values of meteorological quantities in desert areas, Arctic areas, etc.

Traditional approach (stationary sensors) vs. UAVs. The traditional approach to increasing data coverage in an area is to place additional sensors in this area. However, placing a sensor in a remote uninhabited area is costly. On top of the cost of placing the sensors, we also need to organize periodic maintenance of the sensors, which leads to additional expenses - and the corresponding budgets are limited.

Stationarily placed sensors provide us only with the information about a small vicinity of their location. It is therefore desirable, instead, to use measuring instruments that would provide a much wider area coverage. Since stationary devices cannot provide a wide coverage, we need mobile devices. In sparsely populated areas, with few (or even none) roads, this means that we need to use aerial devices. Thus, it is desirable to use Unmanned Aerial Vehicles (UAVs).

Use of UAVs in meteorology: current experience and future plans. Preliminary experience shows that the UAVs can indeed bring a lot of useful meteorological information; see, e.g., [4], [5]. 
At present, at least in the US, the UAVs are not actively used to collect meteorological data, because until Fall 2015, there are very strict regulations in place enforced by the Federal Aviation Administration. These regulations make the use of UAVs for simple tasks (like collecting meteorological data) too complicated and too expensive.

However, in view of the numerous potential advantages of UAVs, the US Congress has required the FAA to adopt, by Fall 2015, more relaxed regulations that would encourage the use of UAVs. In preparation for this, many researchers are developing techniques and methods for using UAVs.

Other potential applications of UAVs. There are many other potential applications of UAVs as mobile sensors. For example, they can be used, instead of expensive stationary sensors, to monitor traffic and thus, to be able to estimate the driver's paths that would minimize city traffic congestions. They can be used to monitor potential areas of border drug smuggling; see, e.g., [10], etc.

Need for deploying swarms of UAVs. In most cases, to get a better coverage, we need to deploy several UAVs. For example, we need several UAVs to collect meteorological information in a large area, we need several UAVs to monitor traffic in a big city, we need several UAVs to successfully monitor for potential drug smuggling attempts along a long stretch of the border, etc.

Jointly, these UAVs should cover a large area. Thus, the subareas covered by individual UAVs should intersect. It is therefore desirable for these UAVs to coordinate their activities, to avoid duplications, and - what would be even worse interference and collisions between the UAVs operating close to each other. Such a group of mutually coordinated UAVs is usually called a swarm.

Swarms of UAVs: need to use intelligent (fuzzy) control. One of the main challenges in operating a swarm of UAVs is to provide the corresponding control.

For individual UAVs, it is possible to come up with optimal control strategies; see, e.g., [4], [5], [10]. However, already these individual control problems are not easy to formulate and to solve.

For a swarm of UAVs, we need to determine the coordinates and velocities of all the UAVs. The sheer number of unknowns makes the corresponding optimization problem too complicated to hope for an explicit solution. A reasonable alternative is thus to utilize expert rules - namely, to use fuzzy control methodology (the methodology specifically designed to translate such rules into an exact control strategy) to translate these rules into an exact strategy for controlling a swarm of UAVs.

Use of fuzzy control for a swarm of UAVs: challenges. To implement the above idea, we need to elicit the corresponding rules from the experts. These rules have to describe what control to apply in each situation, depending on the current locations and velocities of all the UAVs in the swarm.

And here lies a problem. Such a rule elicitation can be feasibly done in situations when the desired control depends on the value of two (or, more generally, a few) quantities. We just select five or so possible fuzzy values of each of these quantities (small, medium, etc.), and then ask the expert what control to apply for each pair of such values. For example, for a robot trying to avoid an obstacle we can ask what control to apply if the robot's distance to the obstacle is small and its velocity is medium, what to do when the distance is large and the velocity is small, etc. This is how fuzzy rules are usually elicited, and an expert can usually generate the corresponding $5 \times 5=25$ (or fewer) rules.

For a swarm, however, in principle, the control applied to each UAV may depend on the locations and velocities of all the other UAVs. Even if use three levels of each of $N$ variable, then for large $N$, the number of resulting combinations $\left(3^{N}\right)$ is huge; it is not realistically possible to ask that many questions to the experts.

What we do in this paper. In this paper, we show that, in general, it is possible to use model reduction techniques to decrease the number of questions and thus, to make rules elicitation possible. In addition to general results, we also show that for the UAVs, optimal control indeed leads to a model reduction - and thus, to a drastic decrease in the corresponding number of questions.

\section{Model Reduction: General Idea And Its PHYSICAL AND MATHEMATICAL FOUNDATIONS}

The main idea behind Newton's physics: reminder. In order to describe the main ideas behind model reduction, let us recall that the foundations of most of modern physics is still Newton's physics.

In Newton's physics, the state $s$ of an object is described by its location $\vec{x}$ and its velocity $\vec{v}: s=(\vec{x}, \vec{v})$. To describe how the state changes with time, we need to describe how the coordinates and the velocities change. The change in coordinates is determined by the velocities: $\frac{d \vec{x}}{d t}=\vec{v}$, while the change in the velocity - i.e., acceleration $\vec{a}=\frac{d \vec{v}}{d t}-$ is determined by the Second Newton's law $m \cdot \vec{a}=\vec{F}$, where $m$ is the object's mass, and $\vec{F}$ is the sum $\vec{F}=\sum_{i} \vec{F}_{i}$ of all the forces that act on this body.

By dividing both sides of Newton's equation by the mass $m$, we can conclude that $\vec{a}=\sum_{i} \vec{f}_{i}$, where we denoted $\vec{f}_{i} \stackrel{\text { def }}{=} \frac{1}{m} \cdot \vec{F}_{i}$. In other words, for the state $s$, we have $\frac{d s}{d t}=\left(\vec{v}, \sum_{i} \overrightarrow{f_{i}}\right)$, i.e., $\frac{d s}{d t}=\sum_{i} g_{i}$, where $g_{i}$ denote the terms corresponding to different forces.

To describe the dynamics, we therefore need to know what type of forces are possible. Some of these forces are determined by the state $s$ of the object itself. For example, when the object is moving through atmosphere or through water, it is slowed down by the friction force which, in general, depends on the object's velocity $\vec{F}_{0}=F(\vec{v})$. the corresponding term $\overrightarrow{f_{0}}$ thus also depends only on the state of the given object. In this case, we have $\frac{d s}{d t}=g(s)$. 
Other forces describe the action of other objects on this object. A typical example of such a force - the main one described by Newton himself - is the force of gravity, which describes the general effect of all other bodies as

$$
\vec{F}=\sum_{i} G \cdot \frac{m \cdot m_{i}}{\left|\vec{x}-\vec{x}_{i}\right|^{3}} \cdot\left(\vec{x}_{i}-\vec{x}\right),
$$

which corresponds to

$$
\vec{f}=\frac{1}{m} \cdot \vec{F}=\sum_{i} G \cdot \frac{m_{i}}{\left|\vec{x}-\vec{x}_{i}\right|^{3}} \cdot\left(\vec{x}_{i}-\vec{x}\right) .
$$

The resulting force is the sum of the terms depending on the state $s$ of the current object and the states $s_{i}$ of other objects: $\frac{d s}{d t}=\sum_{i} g\left(s, s_{i}\right)$.

In principle, we can also have forces corresponding to the combined effect of several other objects, in which case we have

$$
\vec{F}=\sum_{i, j} \vec{F}\left(s, s_{i}, s_{j}\right)+\sum_{i, j, k} \vec{F}\left(s, s_{i}, s_{j}, s_{k}\right)+\ldots,
$$

so that

$$
\frac{d s}{d t}=\sum_{i, j} g\left(s, s_{i}, s_{j}\right)+\sum_{i, j, k} g\left(s, s_{i}, s_{j}, s_{k}\right)+\ldots
$$

By grouping together terms corresponding to different number of affecting objects, we get the general description of the state's dynamics as described by Newton's laws:

$$
\begin{gathered}
\frac{d s}{d t}=g(s)+\sum_{i} g\left(s, s_{i}\right)+\sum_{i, j} g\left(s, s_{i}, s_{j}\right)+ \\
\sum_{i, j, k} g\left(s, s_{i}, s_{j}, s_{k}\right)+\ldots
\end{gathered}
$$

The corresponding mathematical model and the main idea behind model reduction. In general, the right-hand side of the above formula describes a function of many variables $s$, $s_{1}, s_{2}, \ldots$ The above representation presents this function as a sum of different terms of increased complexity: first come terms which depend only on a single state $s$, then terms that depend on two states $s$ and $s_{i}$, then terms that depend in three states $s, s_{i}$, and $s_{j}$, etc.

This formula inspired mathematicians to propose representing a general function of many variables in a similar form (see, e.g., [19], [25]):

$$
\begin{gathered}
f\left(x_{1}, \ldots, x_{n}\right)=f_{0}+\sum_{i} f_{i}\left(x_{i}\right)+\sum_{i, j} f_{i, j}\left(x_{i}, x_{j}\right)+ \\
\sum_{i, j, k} f_{i, j, k}\left(x_{i}, x_{j}, x_{k}\right)+\ldots
\end{gathered}
$$

This representation is known as model reduction (or, alternatively, high dimensional model representation (HDMR)), because in many practical situations, a few first terms in this representation are sufficient to reasonably accurately represent a function.
In most cases, it is sufficient to use the first three terms

$$
f\left(x_{1}, \ldots, x_{n}\right) \approx f_{0}+\sum_{i} f_{i}\left(x_{i}\right)+\sum_{i, j} f_{i, j}\left(x_{i}, x_{j}\right) .
$$

Beyond Newton's physics: how relativity theory makes many physical formulas and related computations simpler. It is well known that, in spite of the fact that most phenomena of macroscopical physics are well described by Newton's physics, in general, Newton's physics is only approximately true. Historically the first deviation from Newton's physics came with Relativity Theory; see, e.g., [7].

At first glance, the equations of special relativity are somewhat more complicated than the equations of Newton's mechanics. As a result, e.g., the need to take into account relativistic effects in celestial mechanics makes the corresponding computations much more complex; see, e.g., [11], [14].

However, there is a fundamental feature of relativity theory that makes computations simpler. Namely, in Newton's physics, all interactions were interactions-at-distance. As a result, to predict the future state of an object, theoretically, we need to know the current state of all other objects in the Universe - since in principle, each of these other objects, no matter how distant they are, can affect the state of the given object.

In contrast, in relativity theory, the speed of all interactions and all interactions is limited by the speed of light $c$. Thus, in order to predict, based on the information available at time $t$, the state of an object at location $\vec{x}$ at moment $t+\Delta$, it is sufficient to take into account only the objects whose distance from $\vec{x}$ does not exceed $c \cdot \Delta t$. This limitation makes predictions easier to compute.

How seeming action-at-a-distance is explained in relativistic physics. If there is no action-at-a-distance, then how can we explain interaction between distant bodies?

The explanation is straightforward, and it comes from observing phenomena when the details of such seeming actionat-a-distance can be analyzed in detail. When one person talks to another one, what happens in that molecules of air start interacting, and the corresponding acoustic wave travels from the first person's mouth to the second person's ears. When distant insects find each other during the mating season, what happens is that molecules of pheromones travel from one insect to another. When one person calls another person's cell phone, the electromagnetic waves - i.e., photons - travel from one cell phone to the other.

In general, in relativity theory, a seeming action-at-adistance is always explained as an exchange of some auxiliary objects.

Historical comment. It is worth mentioning that A. Einstein was awarded his Nobel prize not for the Relativity theory which at that time was mostly of theoretical interest - but for his analysis of the physics of photons, practically important analysis that was clearly motivated by the theoretical ideas behind his relativity theory. 
How local character of interactions can help us. In Newton's physics, when action-at-a-distance is possible, we can, in principle, envision a joint effect of three, four, and more objects. As a result, in the general formula for model reduction, we could have terms of the type $f\left(x_{i}, x_{j}, x_{k}\right), f\left(x_{i}, x_{j}, x_{k}, x_{\ell}\right)$, etc.

In contrast, in relativistic physics, while an interaction between two objects is quite possible - when these objects happen to be sufficiently close - interaction between three objects is highly improbable, since it is highly improbably that three objects accidentally happen to be in the same location at the same moment of time.

This impossibility can be illustrated on the example of chemical kinetics: while it sometimes feels like some chemical reactions transform three different molecules into something new, it always turns out that only two molecules can interact at each moment of time (since the probability of three molecules accidentally meeting is miniscule). What actually happens is that two molecules interact, and then the result of this interaction interacts with the third molecule. This is, for example, what usually happens during catalysis, when an additional molecule (called a catalyst) speeds up the reaction between the two original molecules; see, e.g., [1], [2], [3], [15], [16].

Since only pair-wise interactions are thus physically possible, we can use only pair-wise interaction terms in the general description of physically meaningful functions

$$
f\left(x_{1}, \ldots, x_{n}\right)=f_{0}+\sum_{i} f_{i}\left(x_{i}\right)+\sum_{i, j} f_{i, j}\left(x_{i}, x_{j}\right),
$$

and in the corresponding description of a system's dynamics:

$$
\frac{d s}{d t}=g(s)+\sum_{i} g\left(s, s_{i}\right)+\sum_{i, j} g\left(s, s_{i}, s_{j}\right) \text {. }
$$

Historical comment. It is worth mentioning that, while in principle, Newton's theory allows triple, quadruple, etc. interactions, the main success of the original Newton's theory celestial mechanics - used only pairwise interactions.

Mathematical comment. This physical result is in good accordance with the famous Kolmogorov's theorem that any continuous function can be represented as a composition of functions of one and two variables [13] (see also [26]).

This theorem was proven as part of the work on the famous Hilbert's problems [6], [9] that David Hilbert, the most famous mathematician in the year 1900, selected as the most important problems for the 20 century mathematics. One of these problems was to prove that not every function of many variables can be represented as a composition of functions of one or two variables - a conjecture that Kolmogorov disproved.

Kolmogorov's result has been used in many application areas, including fuzzy and neural techniques [8], [17], [18], [21], [22], [23]: for example, it explains why in fuzzy logic, we concentrate on unary and binary operations such as negation operations, "and" and "or"-operations (t-norms and tconorms), etc.: all other operations can be represented as compositions of unary and binary ones.
Conclusion about UAVs. We should look for controls that only take into account pair-wise interaction between the UAVs.

Let us show that for swarms of UAVs, this restriction can be explained by the need for optimal control.

\section{NEED FOR OPtiMAl CONTROL EXPlains RESTRICTION TO PAIRWISE COORDINATION BETWEEN UAVS}

Main objectives of a swarm of UAVs: reminder. Our goal is to select the trajectories of all the UAVs in such a way that between them, the UAVs cover the desired area.

What should we optimize. While the UAV is in the air, it automatically measure the values of the corresponding quantities. Due to energy limitations, a UAV cannot be in the air all the time - unless we are talking about a solar-panelled UAV flying over sunny desert; so, we need to refuel it. The longer the UAV is in the air, the less refuelling maintenance the system needs.

Thus, when planning UAV trajectories, it is reasonable to maximize the time that the UAV is in the air - i.e., in other word, to minimize the UAV's fuel consumption.

\section{How to minimize UAV's fuel consumption: inertia can help.} How can we minimize the UAVs' fuel consumption? For that, we can use basic physics, namely, again Newton's physics.

In the previous section, we used the Second Newton's Law, now we will use the First Newton's Law - that when no forces act on a body, it retains its speed and the direction of its motion. Of course, the UAV still needs some energy to maintain its flight, to overcome gravity forces that drag it down and friction forces that slow it down. However, in general, in accordance with the First Newton's Law, the fewer deviations of the trajectory from a straight line, the smaller amount of energy needed to support this trajectory.

So, optimal trajectories should consist of several straightline segments, with as few turns as possible. For the fixed amount of fuel, a UAV can fly, in general, a trajectory of a fixed overall length. We want to minimize the number of turns during this length; this is equivalent to maximizing the length of each straight-line segment.

So, if we need to design a swarm of UAVs to cover an area of size $W \times L$, with $W \leq L$, it makes sense to have all straightline trajectory segments to be of the maximum possible length within this region - i.e., of length $L$. Thus, each UAV should go from one side of the region to another and then back again, covering the area of size $w \cdot L$, where $w$ is how much width this UAV can cover before it runs out of fuel; see Fig. 1.

Such long vertical regions are neighboring to each other, so each UAV needs to coordinate its motion only with two immediate neighbors: one to the left and one to the right.

This conclusion justifies, for the case of UAVs, our general conclusion that only pair-wise interactions need to be take into account - and this open the possibility of eliciting fuzzy rules for controlling swarms of UAVs. 


\section{ACKNOWLEDGMENTS}

This work was supported in part by the National Science Foundation grants HRD-0734825 and HRD-1242122 (CyberShARE Center of Excellence) and DUE-0926721.

$w$

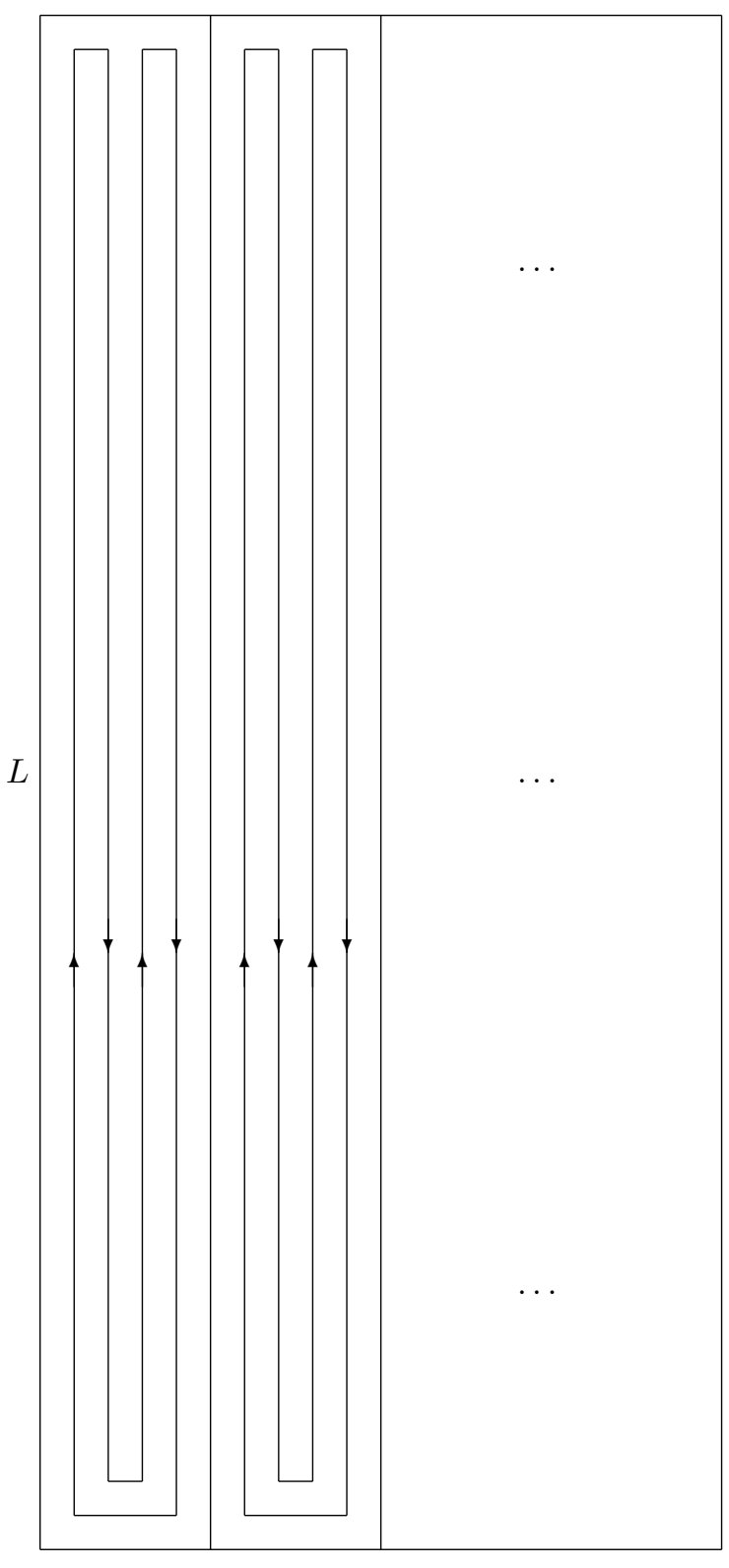

$W$

Figure 1: Optimal trajectories

\section{REFERENCES}

[1] N. R. Amundson and R. Aris, Mathematical Methods in Chemical Engineering, Prentice Hall, Englewood Cliffs, New Jersey, 1973.

[2] R. Aris, Mathematical Modeling Techniques, Pitman, London, 1978.

[3] R. Aris, Elementary Chemical Reactor Analysis, Dover Publ., New York, 2000.
[4] J. Brady, O. Lerma, V. Kreinovich, and C. Tweedie, Toward computing an optimal trajectory for an environment-oriented Unmanned Aerial Vehicle (UAV) under uncertainty, Journal of Uncertain Systems, 9, 2015, to appear.

[5] J. Brady, O. Lerma, V. Kreinovich, C. Tweedie, and R. Romero, Toward computing an optimal trajectory for an environment-oriented Unmanned Aerial Vehicle (UAV), Extended Abstracts of the NSF Joint Annual Meeting of the Centers of Research Excellence in Science and Technology (CREST) Historically Black Colleges and Universities - Research Infrastructure for Science and Engineering (HBCU-RISE) CREST/HBCURISE-JAM'2010, Washington, DC, June 6-9, 2010, pages 69-70.

[6] F. E. Browder (ed.), Mathematical Developments Arising from Hilbert's Problems, American Mathematical Society, Providence, Rhode Island, 1976.

[7] R. Feynman, R. Leighton, and M. Sands, The Feynman Lectures on Physics, Addison Wesley, Boston, Massachusetts, 2005.

[8] R. Hecht-Nielsen, Kolmogorov's mapping neural network existence theorem, Proceedings of the IEEE International Conference on Neural Networks, San Diego, SOS Printing, 1987, Vol. 2, pages 11-14.

[9] D. Hilbert, Mathematical problems, lecture delivered before the International Congress of Mathematics in Paris in 1900, Bulletin of the American Mathematical Society, 8:437-479, 1902.

[10] C. Kiekintveld, V. Kreinovich, and O. Lerma, Optimizing trajectories for Unmanned Aerial Vehicles (UAVs) patrolling the border, In: R. R. Yager, M. Z. Reformat, S. N. Shahbazova, and S. Ovchinnikov (eds.), Proceedings of the World Conference on Soft Computing, San Francisco, CA, May 23-26, 2011.

[11] S. Klioner, P. K. Seidelmann, and M. H. Soffel (eds.), Relativity in Fundamental Astronomy, Proceedings of IAU Symposium No. 261, Cambridge University Press, Cambridge, UK, 2009.

[12] G. J. Klir and B. Yuan, Fuzzy Sets and Fuzzy Logic: Theory and Applications, Prentice Hall, Upper Saddle River, New Jersey, 1995.

[13] A. N. Kolmogorov, On the representation of continuous functions of several variables by superposition of continuous functions of one variable and addition, Dokl. Akad. Nauk SSSR, 114:369-373, 1957.

[14] V. Kreinovich, Astronomical tests of relativity: beyond Parameterized Post-Newtonian Formalism (PPN), to testing fundamental Principles, In: S. Klioner, P. K. Seidelmann, and M. H. Soffel (eds.), Relativity in Fundamental Astronomy, Proceedings of IAU Symposium No. 261, Cambridge University Press, Cambridge, UK, 2009, pages 56-61.

[15] V. Kreinovich, All kinds of behavior are possible in chemical kinetics: a theorem and its potential applications to chemical computing, In: E. Katz (ed.), Molecular and Supramolecular Information Processing: From Molecular Switches to Logical Systems, Wiley-VCH, Wienheim, Germany, pages 237-258, 2012.

[16] V. Kreinovich and O. Fuentes, High-concentration chemical computing techniques for solving hard-to-solve problems, and their relation to numerical optimization, neural computing, reasoning under uncertainty, and freedom of choice, In: E. Katz (ed.), Molecular and Supramolecular Information Processing: From Molecular Switches to Logical Systems, Wiley-VCH, Wienheim, Germany, pages 209-235, 2012.

[17] V. Kurkova, Kolmogorov's Theorem is relevant, Neural Computation, 3:617-622, 1991.

[18] V. Kurkova, Kolmogorov's Theorem and multilayer neural networks, Neural Networks, 5:501-506, 1992.

[19] G. Li, S.-W. Wang, and H. Rabitz, High Dimensional Model Representation (HDMR): concepts and applications, Proceedings of the Institute of Mathematics and Its Applicatons Workshop on Atmospheric Modeling, Minneapolis, Minnesota, March 15-19, 2000.

[20] E. N. Lorenz, Deterministic nonperiodic flow, Journal of the Atmospheric Sciences 20(2):130-141, 1963.

[21] M. Nakamura, R. Mines, and V. Kreinovich, Guaranteed intervals for Kolmogorov's theorem (and their possible relation to neural networks), 3:183-199, Interval Computations, 1993.

[22] H. T. Nguyen and V. Kreinovich, Kolmogorov's Theorem and its impact on soft computing, In: R. R. Yager and J. Kacprzyk, The Ordered Weighted Averaging Operators: Theory and Applications, Kluwer, Boston, MA, pages 3-17, 1997.

[23] H. T. Nguyen, V. Kreinovich, and D. Sprecher, Normal forms for fuzzy logic - an application of Kolmogorov's theorem, International Journal 
on Uncertainty, Fuzziness, and Knowledge-Based Systems, 4(4):331-349, 1996.

[24] H. T. Nguyen and E. A. Walker, A First Course in Fuzzy Logic, Chapman and Hall/CRC, Boca Raton, Florida, 2006.

[25] I. M. Sobol', Sensitivity estimates for nonlinear mathematical models, Mathematical Modeling and Computational Experiment, 1(4):407-414, 1993.

[26] D. A. Sprecher, An improvement in the Superposition Theorem of Kolmogorov, Journal of Mathematical Analysis and Applications, 38:208213, 1972.

[27] L. A. Zadeh, Fuzzy sets, Information and Control, 8:338-353, Elsevier, 1965. 